\title{
A refinement of Nesterenko's linear independence criterion with applications to zeta values
}

\author{
Stéphane Fischler • Wadim Zudilin
}

Received: 9 May 2009 / Published online: 2 December 2009

(C) The Author(s) 2009. This article is published with open access at Springerlink.com

\begin{abstract}
We refine (and give a new proof of) Nesterenko's famous linear independence criterion from 1985, by making use of the fact that some coefficients of linear forms may have large common divisors. This is a typical situation appearing in the context of hypergeometric constructions of $\mathbb{Q}$-linear forms involving zeta values or their $q$-analogs. We apply our criterion to sharpen previously known results in this direction.
\end{abstract}

\section{Introduction}

\subsection{Nesterenko's criterion}

In this text, we refine Nesterenko's linear independence criterion by taking into account the existence of common divisors to the coefficients of the linear forms. Consider the following situation:

The work of W. Zudilin was supported by the Max Planck Institute for Mathematics (Bonn) and the Hausdorff Center for Mathematics (Bonn).

S. Fischler $(\varangle)$

Laboratoire de Mathématiques d'Orsay, Université Paris-Sud, 91405 Orsay Cedex, France

e-mail: Stephane.Fischler@math.u-psud.fr

\section{S. Fischler}

CNRS, 91405 Orsay Cedex, France

\section{W. Zudilin}

School of Mathematical and Physical Sciences, University of Newcastle,

Callaghan, NSW 2308, Australia 
(N) Let $\xi_{0}, \ldots, \xi_{r}$ be real numbers, with $r \geq 1$. Let $0<\alpha<1$ and $\beta>1$. For any $n \geq 1$, let $\ell_{0, n}, \ldots, \ell_{r, n}$ be integers such that

$$
\lim _{n \rightarrow \infty}\left|\sum_{i=0}^{r} \ell_{i, n} \xi_{i}\right|^{1 / n}=\alpha \text { and } \limsup _{n \rightarrow \infty}\left|\ell_{i, n}\right|^{1 / n} \leq \beta \quad \text { for any } i \in\{0, \ldots, r\}
$$

Let us recall a special case of Nesterenko's criterion [14].

Theorem A (Yu. Nesterenko) Assume that hypothesis (N) holds. Then we have

$$
\operatorname{dim}_{\mathbb{Q}} \operatorname{Span}_{\mathbb{Q}}\left(\xi_{0}, \ldots, \xi_{r}\right) \geq 1-\frac{\log \alpha}{\log \beta}
$$

Hypothesis (N) implies $\operatorname{dim}_{\mathbb{Q}} \operatorname{Span}_{\mathbb{Q}}\left(\xi_{0}, \ldots, \xi_{r}\right) \geq 2$, because otherwise $\xi_{0}, \ldots, \xi_{r}$ would be integer multiples of a (possibly zero) real number $\xi$, so that all linear forms $\sum_{i=0}^{r} \ell_{i, n} \xi_{i}$ would be integer multiples of $\xi$. This is impossible since these linear forms tend to 0 without vanishing (for $n$ sufficiently large). This remark shows that the first interesting case is trying to get a dimension greater than or equal to three. This special case of Theorem A reads as follows.

Theorem B Assume that hypothesis (N) holds. If $\alpha \beta<1$, then

$$
\operatorname{dim}_{\mathbb{Q}} \operatorname{Span}_{\mathbb{Q}}\left(\xi_{0}, \ldots, \xi_{r}\right) \geq 3
$$

In other words, among $\xi_{0}, \ldots, \xi_{r}$ there are at least three numbers that are linearly independent over the rationals.

\subsection{A refinement}

We obtain the following improvement of Nesterenko's criterion, the proof of which relies on Minkowski's convex body theorem and yields a new proof of Nesterenko's result.

Theorem 1 Let $\xi_{0}, \ldots, \xi_{r}$ be real numbers, with $r \geq 1$. Let $\tau>0$ and $\gamma_{1}, \ldots, \gamma_{r} \geq 0$. For any $n \geq 1$ and any $i \in\{0, \ldots, r\}$, let $\ell_{i, n} \in \mathbb{Z}$. For $n \geq 1$ and $i \in\{1, \ldots, r\}$, let $\delta_{i, n}$ be a positive divisor of $\ell_{i, n}$ such that

(i) $\delta_{i, n}$ divides $\delta_{i+1, n}$ for any $n \geq 1$ and any $i \in\{1, \ldots, r-1\}$, and

(ii) $\frac{\delta_{j, n}}{\delta_{i, n}}$ divides $\frac{\delta_{j, n+1}}{\delta_{i, n+1}}$ for any $n \geq 1$ and any $0 \leq i<j \leq r$, with $\delta_{0, n}=1$.

Assume that there exists an increasing sequence $\left(Q_{n}\right)_{n \geq 1}$ of integers such that, as $n \rightarrow \infty$, the following conditions are met: 


$$
\begin{aligned}
& Q_{n+1}=Q_{n}^{1+o(1)}, \\
& \max _{0 \leq i \leq r}\left|\ell_{i, n}\right| \leq Q_{n}^{1+o(1)}, \\
& \left|\sum_{i=0}^{r} \ell_{i, n} \xi_{i}\right|=Q_{n}^{-\tau+o(1)}, \\
& \delta_{i, n}=Q_{n}^{\gamma_{i}+o(1)} \text { for any } i \in\{1, \ldots, r\} .
\end{aligned}
$$

Let $s=\operatorname{dim}_{\mathbb{Q}} \operatorname{Span}_{\mathbb{Q}}\left(\xi_{0}, \ldots, \xi_{r}\right)-1$. Then we have

$$
s \geq \tau+\gamma_{1}+\cdots+\gamma_{s}
$$

Taking $Q_{n}=\beta^{n}$ and $\tau=-(\log \alpha) /(\log \beta)$ we obtain the following special case.

Corollary 1 Assume that hypothesis $(\mathbf{N})$ holds. For any $n \geq 1$ and any $i \in\{1, \ldots, r\}$, let $\delta_{i, n}$ be a positive divisor of $\ell_{i, n}$. Assume that

(i) $\delta_{i, n}$ divides $\delta_{i+1, n}$ for any $n \geq 1$ and any $i \in\{1, \ldots, r-1\}$, and

(ii) $\frac{\delta_{j, n}}{\delta_{i, n}}$ divides $\frac{\delta_{j, n+1}}{\delta_{i, n+1}}$ for any $n \geq 1$ and any $0 \leq i<j \leq r$, with $\delta_{0, n}=1$.

Furthermore, assume that for any $i \in\{1, \ldots, r\}$ the limit of $\delta_{i, n}^{1 / n}$ as $n \rightarrow \infty$ exists. Let $s=\operatorname{dim}_{\mathbb{Q}} \operatorname{Span}_{\mathbb{Q}}\left(\xi_{0}, \ldots, \xi_{r}\right)-1$. Then we have $s \geq 1$ and

$$
\alpha \beta^{s} \geq \prod_{i=1}^{s} \lim _{n \rightarrow \infty} \delta_{i, n}^{1 / n} .
$$

The conclusion of this corollary has to be understood as a lower bound for $s$, namely,

$$
s \geq-\frac{\log \left(\alpha / \prod_{i=1}^{s} \lim _{n \rightarrow \infty} \delta_{i, n}^{1 / n}\right)}{\log \beta}
$$

but is should be noted that the product contains $s$ factors.

The following special case of Corollary 1 , in which we let $d_{n}=\operatorname{lcm}(1,2, \ldots, n)$, is useful when studying linear independence of zeta values (see, for example, the proofs of Theorems 3 and 5).

Corollary 2 Assume that hypothesis $(\mathbf{N})$ holds. Let $e_{1} \leq \cdots \leq e_{r}$ be non-negative integers such that $d_{n}^{e_{i}}$ divides $\ell_{i, n}$ for any $n \geq 1$ and any $i \in\{1, \ldots, r\}$.

Let $s=\operatorname{dim}_{\mathbb{Q}} \operatorname{Span}_{\mathbb{Q}}\left(\xi_{0}, \ldots, \xi_{r}\right)-1$. Then we have $s \geq 1$ and

$$
s \geq \frac{e_{1}+\cdots+e_{s}-\log \alpha}{\log \beta} .
$$

Again the lower bound we obtain for $s$ in this corollary actually depends on $s$ itself. 
It is interesting to see what happens with Theorem 1 when we just try to prove that $\operatorname{dim}_{\mathbb{Q}} \operatorname{Span}_{\mathbb{Q}}\left(\xi_{0}, \ldots, \xi_{r}\right) \geq 3$, as in Theorem B. Then we may assume, without loss of generality, that $\delta_{1, n}=\cdots=\delta_{r, n}$ for any $n$. In this case, the Assumptions (i) and (ii) simply mean that $\delta_{1, n}$ divides $\delta_{1, n+1}$. Actually, we obtain the following stronger improvement of Theorem $\mathrm{B}$, in which this assumption is replaced with a lower bound on the greatest common divisor of $\delta_{1, n}$ and $\delta_{1, n+1}$.

Theorem 2 Assume that hypothesis $(\mathbf{N})$ holds. For any $n \geq 1$, let $\delta_{n}$ be a common positive divisor of $\ell_{1, n}, \ldots, \ell_{r, n}$. Assume that

$$
\alpha \beta<\liminf _{n \rightarrow \infty}\left(\operatorname{gcd}\left(\delta_{n}, \delta_{n+1}\right)\right)^{1 / n} .
$$

Then

$$
\operatorname{dim}_{\mathbb{Q}} \operatorname{Span}_{\mathbb{Q}}\left(\xi_{0}, \ldots, \xi_{r}\right) \geq 3
$$

The main interest of Theorem 2 is actually its proof, which is simpler than that of Theorem 1 (cf. Sects. 2.2 and 2.3).

\subsection{Applications}

Theorem 1 is interesting whenever Nesterenko's linear independence criterion is used and the coefficients of the linear forms are known to have common divisors. Among the various situations where this phenomenon appears, we have chosen an arithmetic problem for the so-called odd zeta values - the values of Riemann's zeta function

$$
\zeta(l)=\sum_{k=1}^{\infty} \frac{1}{k^{l}}
$$

at odd integers $l>1$; see $[1,2,7,11,20,22]$ for history and known results in this arithmetic direction. The following theorem improves on previous bounds $\left(i_{1} \leq 145\right.$ and $i_{2} \leq 1971$ ) from [20, Theorem 0.3].

Theorem 3 There exist odd integers $i_{1} \leq 139$ and $i_{2} \leq 1961$ such that the numbers

$$
1, \zeta(3), \zeta\left(i_{1}\right), \text { and } \zeta\left(i_{2}\right)
$$

are linearly independent over $\mathbb{Q}$.

Another but related application is devoted to arithmetic properties of the following $q$-analog of Riemann's zeta function $(|q|<1)$ :

$$
\zeta_{q}(l)=\sum_{k=1}^{\infty} \frac{k^{l-1} q^{k}}{1-q^{k}}=\sum_{k=1}^{\infty} \sigma_{l-1}(k) q^{k},
$$


where $\sigma_{l-1}(k)=\sum_{d \mid k} d^{l-1}$. A usual setup for a number $q$ is to be of the form $1 / p$, where $p \in \mathbb{Z} \backslash\{0, \pm 1\}$. Although the irrationality of $\zeta_{q}(1)$ and even the transcendence of $\zeta_{q}(l)$ for any even positive integer $l$ are known, not so much is obtained for $\zeta_{q}(l)$ with $l>1$ odd; we refer the reader to the works $[10,12]$ for details. For example, Jouhet and Mosaki [10] show that at least one of the four numbers $\zeta_{q}(3), \zeta_{q}(5), \zeta_{q}(7)$, $\zeta_{q}(9)$ is irrational and give further results for the odd $q$-zeta values in the spirit of Theorem 3. Our next theorem sharpens slightly the corresponding bounds on $i_{1}, i_{2}$ and $i_{3}$ proved in [10].

Theorem 4 Let $q$ be a rational of the form $1 / p$, where $p \in \mathbb{Z} \backslash\{0, \pm 1\}$. There exist odd integers $1<i_{0}<i_{1}<i_{2}<i_{3}$ such that $i_{0} \leq 9, i_{1} \leq 37, i_{2} \leq 83, i_{3} \leq 145$ and the numbers

$$
1, \zeta_{q}\left(i_{0}\right), \zeta_{q}\left(i_{1}\right), \zeta_{q}\left(i_{2}\right), \text { and } \zeta_{q}\left(i_{3}\right)
$$

are linearly independent over $\mathbb{Q}$.

Our third application also appeals to arithmetic of the odd zeta values, but this time we add $\log 2$ to the set.

Theorem 5 There exist odd integers $i_{1} \leq 93$ and $i_{2} \leq 1151$ such that the numbers

$$
1, \log 2, \zeta\left(i_{1}\right), \text { and } \zeta\left(i_{2}\right)
$$

are linearly independent over $\mathbb{Q}$.

In our proof of Theorem 5 we use a (seemingly) new hypergeometric construction of linear forms in $1, \log 2$ and odd zeta values. We find rather curious that a 'degenerate' case of our construction, when odd zeta values do not occur at all, resembles well the rational approximations [1] from Apéry's proof of the irrationality of $\zeta$ (3) (cf., for example, [7]); this is the subject of our final Sect. 3.4.

\section{The linear independence criterion}

In this part, we prove (Sect. 2.1) our main linear independence criterion (namely Theorem 1 stated in Sect. 1), as well as its special case (Sect. 2.2) corresponding to Theorem 2. We gather some remarks in Sect. 2.3.

\subsection{Proof of the criterion}

Let us start with two remarks.

Remark 1 The existence of arbitrarily small non-zero linear combinations of $\xi_{0}, \ldots, \xi_{r}$ with integer coefficients implies $\operatorname{dim}_{\mathbb{Q}} \operatorname{Span}_{\mathbb{Q}}\left(\xi_{0}, \ldots, \xi_{r}\right) \geq 2$, that is, $s \geq 1$. 
Remark 2 In the statement of Theorem 1 and in all other linear independence criteria we prove in this text, no assumption is made on whether $\xi_{0}$ vanishes or not. Actually, we can always assume that $\xi_{0} \neq 0$, because if $\xi_{0}=0$ then Remark 1 provides an integer $i$ such that $\xi_{i} \neq 0$, and we can consider the linear forms $0 \xi_{i}+\ell_{1, n} \xi_{1}+\cdots+\ell_{r, n} \xi_{r}$ in $\left(\xi_{i}, \xi_{1}, \ldots, \xi_{r}\right)$.

The proof of Theorem 1 splits into two parts. To begin with, let us prove this result under the assumption that $\xi_{0}, \ldots, \xi_{r}$ are linearly independent over the rationals (that is, $s=r)$.

Denote by $\xi$ the point $\left(\xi_{0}, \ldots, \xi_{r}\right) \in \mathbb{R}^{r+1}$, and by $L_{n}$ the linear form $\ell_{0, n} X_{0}+$ $\cdots+\ell_{r, n} X_{r}$, so that $L_{n}(\xi)=\sum_{i=0}^{r} \ell_{i, n} \xi_{i}$.

Thanks to Remark 2, we may assume that $\xi_{0} \neq 0$ and even $\xi_{0}=1$ (dividing all $\xi_{i}$ by $\xi_{0}$ if necessary).

Let $n$ be a sufficiently large integer. In what follows, $o(1)$ stands for any sequence that tends to 0 as $n$ tends to infinity.

We take

$$
R_{n}=\frac{\delta_{r, n}}{2\left|L_{n}(\xi)\right|} \text { and } \varepsilon_{n}=\delta_{r, n}\left(\frac{3\left|L_{n}(\xi)\right|}{\prod_{i=1}^{r} \delta_{i, n}}\right)^{1 / r}
$$

so that

$$
\varepsilon_{n}=Q_{n}^{\gamma_{r}-\left(\tau+\gamma_{1}+\cdots+\gamma_{r}\right) / r+o(1)}
$$

Arguing by contradiction, assume that $\tau+\gamma_{1}+\cdots+\gamma_{r}>r$. Consider the set $\mathcal{C}_{n}$ consisting in all $\left(q_{0}, \ldots, q_{r}\right) \in \mathbb{R}^{r+1}$ such that

$$
\left|q_{0}\right| \leq \frac{R_{n}}{\delta_{r, n}} \quad \text { and, for any } i \in\{1, \ldots, r\}, \quad\left|\delta_{i, n} q_{0} \xi_{i}-q_{i}\right| \leq \frac{\delta_{i, n}}{\delta_{r, n}} \varepsilon_{n} .
$$

The volume of $\mathcal{C}_{n}$ is

$$
\frac{2^{r+1} R_{n} \varepsilon_{n}^{r} \prod_{i=1}^{r} \delta_{i, n}}{\delta_{r, n}^{r+1}}=\frac{3}{2} \cdot 2^{r+1}>2^{r+1} .
$$

Moreover $\mathcal{C}_{n}$ is convex. To prove this fact, let $\left(q_{0}, \ldots, q_{r}\right)$ and $\left(q_{0}^{\prime}, \ldots, q_{r}^{\prime}\right)$ be elements of $\mathcal{C}_{n}$, and let $\lambda \in[0,1]$. Let $q_{i}^{\prime \prime}=\lambda q_{i}+(1-\lambda) q_{i}^{\prime}$ for any $i \in\{0, \ldots, r\}$. Then $\left|q_{0}^{\prime \prime}\right| \leq \lambda\left|q_{0}\right|+(1-\lambda)\left|q_{0}^{\prime}\right| \leq R_{n} / \delta_{r, n}$ and, for any $i \in\{1, \ldots, r\},\left|\delta_{i, n} q_{0}^{\prime \prime} \xi_{i}-q_{i}^{\prime \prime}\right| \leq$ $\lambda\left|\delta_{i, n} q_{0} \xi_{i}-q_{i}\right|+(1-\lambda)\left|\delta_{i, n} q_{0}^{\prime} \xi_{i}-q_{i}^{\prime}\right| \leq \varepsilon_{n} \delta_{i, n} / \delta_{r, n}$. Therefore $\mathcal{C}_{n}$ is a convex body, symmetric with respect to the origin: Minkowski's theorem shows that there is a non-zero integer point $\left(q_{0, n}, \ldots, q_{r, n}\right)$ in $\mathcal{C}_{n}$. Then rescaling

$$
p_{0, n}=\delta_{r, n} q_{0, n} \text { and } p_{i, n}=\frac{\delta_{r, n}}{\delta_{i, n}} q_{i, n} \in \mathbb{Z} \text { for any } i \in\{1, \ldots, r\}
$$


we have $p_{0, n}, \ldots, p_{r, n} \in \mathbb{Z}$ thanks to Assumption (i), and also by definition of $\mathcal{C}_{n}$ :

$$
\left|p_{0, n}\right| \leq R_{n} \text { and }\left|p_{0, n} \xi_{i}-p_{i, n}\right| \leq \varepsilon_{n} \quad \text { for any } i \in\{1, \ldots, r\}
$$

Let $k_{n}$ denote the least positive integer such that

$$
\left|p_{0, n}\right| \leq \frac{\delta_{r, k_{n}}}{2\left|L_{k_{n}}(\xi)\right|}
$$

By definition of $R_{n}$ and thanks to the upper bound (1) on $\left|p_{0, n}\right|, k_{n}$ exists and $k_{n} \leq n$; this upper bound will be used later.

Since $\delta_{r, n}$ divides $\ell_{r, n}$, we have $\gamma_{r} \leq 1$. The assumption $\tau+\gamma_{1}+\cdots+\gamma_{r}>r$ and the definition of $\varepsilon_{n}$ yield $\lim _{n \rightarrow \infty} \varepsilon_{n}=0$. Thanks to (1), this implies $\lim _{n \rightarrow \infty}\left(p_{0, n} \xi_{1}-\right.$ $\left.p_{i, n}\right)=0$. Now $1, \xi_{1}, \ldots, \xi_{r}$ are assumed to be linearly independent, so that $\xi_{1} \notin \mathbb{Q}$ and $\lim _{n \rightarrow \infty}\left|p_{0, n}\right|=+\infty$. The definition of $k_{n}$ then implies $\lim _{n \rightarrow \infty} k_{n}=+\infty$, so that $\delta_{r, k_{n}}=Q_{k_{n}}^{\gamma_{r}+o(1)}$ and $\left|L_{k_{n}}(\xi)\right|=Q_{k_{n}}^{-\tau+o(1)}$.

By minimality of $k_{n}$, we have

$$
\frac{\delta_{r, k_{n}-1}}{2\left|L_{k_{n}-1}(\boldsymbol{\xi})\right|}<\left|p_{0, n}\right| \leq \frac{\delta_{r, k_{n}}}{2\left|L_{k_{n}}(\boldsymbol{\xi})\right|}
$$

with $\delta_{r, k_{n}-1}=Q_{k_{n}-1}^{\gamma_{r}+o(1)}=Q_{k_{n}}^{\gamma_{r}+o(1)}$ and, in the same way, $\left|L_{k_{n}-1}(\xi)\right|=Q_{k_{n}}^{-\tau+o(1)}$. Finally we obtain

$$
\left|p_{0, n}\right|=Q_{k_{n}}^{\gamma_{r}+\tau+o(1)}
$$

Now we can write

$$
\sum_{i=0}^{r} \ell_{i, k_{n}} p_{i, n}=p_{0, n} \sum_{i=0}^{r} \ell_{i, k_{n}} \xi_{i}+\sum_{i=0}^{r} \ell_{i, k_{n}}\left(p_{i, n}-p_{0, n} \xi_{i}\right)
$$

On the right-hand side, the first term has absolute value equal to $\left|p_{0, n} L_{k_{n}}(\xi)\right|$, therefore less than or equal to $\frac{1}{2} \delta_{r, k_{n}}$ by definition of $k_{n}$. Assume the second term has absolute value less than the first one. Then the absolute value of the right-hand side is less than $\delta_{r, k_{n}}$. But it is equal to the left-hand side, which is an integer multiple of $\delta_{r, k_{n}}$, since

$$
\ell_{i, k_{n}} p_{i, n}=\ell_{i, k_{n}} \frac{\delta_{r, n}}{\delta_{i, n}} q_{i, n} \quad \text { is an integer multiple of } \quad \delta_{i, k_{n}} \frac{\delta_{r, k_{n}}}{\delta_{i, k_{n}}}=\delta_{r, k_{n}}
$$

(by condition (ii) and $n \geq k_{n}$ ): it has to be zero. But then both terms on the righthand side would have the same absolute value, in contradiction with the assumption. Therefore we have proved that the second term on the right-hand side of (3) has absolute value greater than or equal to the absolute value of the first one. Combining this 
inequality with (1) and (2) we obtain

$$
Q_{k_{n}}^{\gamma_{r}+o(1)}=\left|p_{0, n} L_{k_{n}}(\xi)\right|=\left|p_{0, n} \sum_{i=0}^{r} \ell_{i, k_{n}} \xi_{i}\right| \leq\left|\sum_{i=0}^{r} \ell_{i, k_{n}}\left(p_{i, n}-p_{0, n} \xi_{i}\right)\right| \leq Q_{k_{n}}^{1+o(1)} \varepsilon_{n} .
$$

Now recall that $k_{n} \leq n$ and $\gamma_{r} \leq 1$; the previous estimate yields

$$
1 \leq Q_{k_{n}}^{1-\gamma_{r}+o(1)} \varepsilon_{n} \leq Q_{n}^{1-\gamma_{r}+o(1)} \varepsilon_{n}=Q_{n}^{1-\left(\tau+\gamma_{1}+\cdots+\gamma_{r}\right) / r+o(1)},
$$

which contradicts the assumption $\tau+\gamma_{1}+\cdots+\gamma_{r}>r$ for $n$ sufficiently large and completes the proof of Theorem 1 under the assumption that $\xi_{0}, \ldots, \xi_{r}$ are linearly independent over the rationals.

Let us now deduce Theorem 1 from this special case.

Because of Assumptions (i) and (ii), we cannot (without loss of generality) change the ordering of $\xi_{0}, \ldots, \xi_{r}$ in order to assume that $\xi_{0}, \ldots, \xi_{s}$ are linearly independent. For this reason we have to focus on the integers $i \in\{1, \ldots, r\}$ such that $\xi_{i}$ does not belong to the $\mathbb{Q}$-vector space spanned by $\xi_{0}, \ldots, \xi_{i-1}$. Let us state this in precise terms.

Thanks to Remarks 1 and 2 , we have $s \geq 1$ and we may assume that $\xi_{0} \neq 0$. Take $i_{0}=0$, and let $i_{1}$ be the least positive integer such that $\xi_{0}$ and $\xi_{i_{1}}$ are linearly independent over the rationals. Define inductively $i_{k}$, for $k \in\{0, \ldots, s\}$, to be the least integer such that $\xi_{i_{0}}, \xi_{i_{1}}, \ldots, \xi_{i_{k}}$ are linearly independent over $\mathbb{Q}$. Clearly, we have $0=i_{0}<i_{1}<\cdots<i_{s}$ and, for any $i \in\{0, \ldots, r\}$, we can write $\xi_{i}$ as a linear combination of those $\xi_{i_{j}}$ with $i_{j} \leq i$. In precise terms, we write $\xi_{i}=\sum_{j=0}^{k} c_{i, j} \xi_{i_{j}}$ with $c_{i, j} \in \mathbb{Q}$ and $k \in\{0, \ldots, s\}$ defined by $i_{k} \leq i<i_{k+1}$ (with $i_{s+1}=r+1$ ). For any $n$, this gives

$$
\sum_{i=0}^{r} \ell_{i, n} \xi_{i}=\sum_{j=0}^{s} \ell_{j, n}^{\prime} \xi_{j}^{\prime}
$$

by letting $\xi_{j}^{\prime}=\xi_{i_{j}}$ and $\ell_{j, n}^{\prime}=\sum_{i=i_{j}}^{r} \ell_{i, n} c_{i, j}$. Let $d$ denote a common denominator of the rational numbers $c_{i, j}$; note that $d$ is independent of $n$. For any $n$ and any $j \in\{0, \ldots, s\}, d \ell_{j, n}^{\prime}$ is an integer and, moreover, a multiple of $\delta_{i_{j}, n}$, since $\delta_{i_{j}, n}$ divides $\delta_{i, n}$ for any $i$ between $i_{j}$ and $r$.

Since $\xi_{0}^{\prime}, \ldots, \xi_{s}^{\prime}$ are linearly independent over the rationals, we can apply to these numbers the special case of Theorem 1 proved at the beginning, with the linear forms $\sum_{j=0}^{s} d \ell_{j, n}^{\prime} \xi_{j}^{\prime}$, the same sequence $\left(Q_{n}\right)_{n \geq 1}$ and the same $\tau$, with divisors $\delta_{j, n}^{\prime}=\delta_{i_{j}, n}$ for $j \in\{1, \ldots, s\}$ and exponents $\gamma_{j}^{\prime}$ which satisfy $\gamma_{j}^{\prime}=\gamma_{i_{j}} \geq \gamma_{j}$, because $\delta_{j, n}^{\prime}=$ $\delta_{i_{j}, n} \geq \delta_{j, n}$ for any $j$ and any $n$. This completes the proof of Theorem 1 .

\subsection{Proof of Theorem 2}

The following statement implies Theorem 2.

Proposition 1 Let $\xi_{0}, \ldots, \xi_{r}$ be real numbers, with $r \geq 1$. For any $n \geq 1$ and any $i \in\{0, \ldots, r\}$, let $\ell_{i, n} \in \mathbb{Z}$. Let $\left(\delta_{n}\right)_{n \geq 1}$ be a sequence of positive integers, such that 
$\delta_{n}$ is a common divisor of $\ell_{1, n}, \ldots, \ell_{r, n}$ for any $n \geq 1$. For any $n \geq 1$, let $H_{n}$ and $\varepsilon_{n}$ be positive real numbers such that

$$
\max _{0 \leq i \leq r}\left|\ell_{i, n}\right| \leq H_{n} \quad \text { and }\left|\sum_{i=0}^{r} \ell_{i, n} \xi_{i}\right| \leq \varepsilon_{n}
$$

Assume that $\sum_{i=0}^{r} \ell_{i, n} \xi_{i} \neq 0$ for infinitely many $n$ and that

$$
\lim _{n \rightarrow \infty} \frac{H_{n} \varepsilon_{n+1}+H_{n+1} \varepsilon_{n}}{\operatorname{gcd}\left(\delta_{n}, \delta_{n+1}\right)}=0 .
$$

Then we have

$$
\operatorname{dim}_{\mathbb{Q}} \operatorname{Span}_{\mathbb{Q}}\left(\xi_{0}, \ldots, \xi_{r}\right) \geq 3
$$

Proof Thanks to Remarks 1 and 2, we have $\operatorname{dim}_{\mathbb{Q}} \operatorname{Span}_{\mathbb{Q}}\left(\xi_{0}, \ldots, \xi_{r}\right) \geq 2$ and we may assume that $\xi_{0} \neq 0$, and even that $\xi_{0}=1$. Since $\xi_{1}, \ldots, \xi_{r}$ play symmetric roles, we may assume that $\xi_{1}$ is irrational. Let us argue by contradiction, assuming on the contrary that $\operatorname{dim}_{\mathbb{Q}} \operatorname{Span}_{\mathbb{Q}}\left(\xi_{0}, \ldots, \xi_{r}\right)=2$. Then $\xi_{2}, \ldots, \xi_{r}$ are rational linear combinations of $\xi_{0}=1$ and $\xi_{1}$. As in the proof of Theorem 1, we obtain a positive integer $d$ independent of $n$ and rational numbers $\ell_{0, n}^{\prime}$ and $\ell_{1, n}^{\prime}$ such that

$$
\sum_{i=0}^{r} \ell_{i, n} \xi_{i}=\ell_{0, n}^{\prime}+\ell_{1, n}^{\prime} \xi_{1}
$$

with $d \ell_{0, n}^{\prime}, d \ell_{1, n}^{\prime} \in \mathbb{Z}$ of absolute value less than $d^{\prime} H_{n}$ for some constant $d^{\prime}$ independent of $n$; moreover, $\delta_{n}$ divides $d \ell_{1, n}^{\prime}$. Now consider the determinant

$$
\Delta_{n}=\left|\begin{array}{cc}
d \ell_{0, n}^{\prime} & d \ell_{1, n}^{\prime} \\
d \ell_{0, n+1}^{\prime} & d \ell_{1, n+1}^{\prime}
\end{array}\right|=d^{2} \ell_{1, n+1}^{\prime}\left(\ell_{0, n}^{\prime}+\ell_{1, n}^{\prime} \xi\right)-d^{2} \ell_{1, n}^{\prime}\left(\ell_{0, n+1}^{\prime}+\ell_{1, n+1}^{\prime} \xi\right)
$$

which satisfies

$$
\left|\Delta_{n}\right| \leq d d^{\prime}\left(H_{n} \varepsilon_{n+1}+H_{n+1} \varepsilon_{n}\right)<\operatorname{gcd}\left(\delta_{n}, \delta_{n+1}\right)
$$

if $n \geq N$ for some integer $N$. Now $\Delta_{n}$ is the determinant of a matrix in which both entries in the second column, namely, $d \ell_{1, n}^{\prime}$ and $d \ell_{1, n+1}^{\prime}$, are integer multiples of $\operatorname{gcd}\left(\delta_{n}, \delta_{n+1}\right)$. Therefore, $\Delta_{n}=0$ for any $n \geq N$. Hence, for any $n \geq N$ the vector $\left(d \ell_{0, n}^{\prime}, d \ell_{1, n}^{\prime}\right)$ is proportional to $\left(d \ell_{0, N}^{\prime}, d \ell_{1, N}^{\prime}\right)$. Now the subgroup $\overline{\mathbb{Z}}^{2} \cap$ $\mathbb{Q}\left(d \ell_{0, N}^{\prime}, d \ell_{1, N}^{\prime}\right)$ of the finitely generated free $\mathbb{Z}$-module $\mathbb{Z}^{2}$ is generated by a single vector, say $\left(u_{0}, u_{1}\right) \in \mathbb{Z}^{2}$. Therefore for any $n \geq N$ there exists an integer $c_{n}$ such that $d \ell_{0, n}^{\prime}=c_{n} u_{0}$ and $d \ell_{1, n}^{\prime}=c_{n} u_{1}$. This implies $\ell_{0, n}^{\prime}+\ell_{1, n}^{\prime} \xi_{1}=c_{n}\left(u_{0}+u_{1} \xi_{1}\right)$ with $c_{n} \in \mathbb{Z}$, in contradiction with the fact that $\ell_{0, n}^{\prime}+\ell_{1, n}^{\prime} \xi_{1}$ tends to 0 without being identically equal to 0 for $n$ sufficiently large, and Proposition 1 follows. 


\subsection{Remarks}

In this section, we make some comments on the proofs given above. In the case where all divisors $\delta_{j, n}$ are equal to 1 , the proof of Theorem 1 gives a new proof of Theorem A, while that of Proposition 1 yet another one in the special case of Theorem B. Nesterenko's general result in [14] is exactly Theorem 1 in the special case $\delta_{j, n}=1$, except for one point: Nesterenko assumes that $Q_{n}^{-\tau_{1}+o(1)} \leq\left|\sum_{i=0}^{r} \ell_{i, n} \xi_{i}\right| \leq Q_{n}^{-\tau_{2}+o(1)}$, whereas we treat the case $\tau_{1}=\tau_{2}$ only. Our method should generalize easily to the situation where $\tau_{1} \neq \tau_{2}$, but we do not write it down because the equality holds in all the applications we have in mind (the point is that knowing the exact size of $\left|\sum_{i=0}^{r} \ell_{i, n} \xi_{i}\right|$ is not really necessary: upper and lower bounds are sufficient to make the arguments work). For the same reason, we did not try to replace $\mathbb{Q}$ with another number field, though Nesterenko's criterion can be generalized to this setting (see $[3,18])$.

Nesterenko's proof consists in obtaining a lower bound for the distance of $\boldsymbol{\xi}=$ $\left(\xi_{0}, \ldots, \xi_{r}\right)$ to any linear subspace of $\mathbb{R}^{r+1}$, defined over $\mathbb{Q}$, of dimension $t<\tau+1$. He proceeds by induction on $t$, whereas we use in the proof of Theorem 1 only the first step (namely $t=1$, see below) of his induction. Colmez [4] writes down Nesterenko's proof in another way (from notes by F. Amoroso). Assume for simplicity that $\xi_{0}, \ldots, \xi_{r}$ are $\mathbb{Q}$-linearly independent (the general case follows from this special case as in the proof of Theorem 1). For any sufficiently large integer $n_{0}$, one constructs by an analogous induction procedure a decreasing sequence $n_{0}>n_{1}>\cdots>n_{r}$ of positive integers such that the determinant $\Delta$ of the matrix $\left[\ell_{i, n_{j}}\right]_{0 \leq i, j \leq r}$ is not zero. The easy case is when $n_{0}, \ldots, n_{r}$ are, roughly speaking, of the same size (for instance, if they are consecutive integers). Then replacing the first line with the linear combination of the lines given by $\left(\xi_{0}, \ldots, \xi_{r}\right)$, we obtain $|\Delta| \leq Q_{n}^{r-\tau+o(1)}$. Since $\Delta$ is a non-zero integer, this gives $r \geq \tau$ and completes the proof in this case. The difficult part of this proof is to obtain some control upon $n_{0}, \ldots, n_{r}$. In Amoroso-Colmez's version of Nesterenko's proof, the sequence $n_{0}>n_{1}>\cdots>n_{r}$ is constructed, and yields the result $r \geq \tau$, but there might be huge gaps between $n_{j}$ and $n_{j+1}$. It would be interesting to know whether such a sequence can always be constructed with $n_{r}$ 'nearly as large' as $n_{0}$; for instance this would enable one to essentially replace the unnatural Assumptions (i) and (ii) of Theorem 1 with: $\delta_{i, n}$ divides $\delta_{i, n+1}$. This is what we do (in the case $r=1$ ) in the proof of Proposition 1 (Sect. 2.2): for infinitely many integers $n_{0}$, we prove that $\Delta \neq 0$ with $n_{1}=n_{0}-1$. This kind of method is similar to the ones used by H. Davenport and W. Schmidt (see, for instance, [5, 6]). This different method is the reason why the assumptions of Proposition 1 are weaker than those of Theorem 1. It is interesting to point out that in Proposition 1 we do not need to assume $Q_{n+1}=Q_{n}^{1+o(1)}$, nor to have a lower bound for $\left|\sum_{i=0}^{r} \ell_{i, n} \xi_{i}\right|$.

On the other hand, our proof of Theorem 1 in Sect. 2.1 relies on a completely different idea. In the case when all divisors $\delta_{j, n}$ are equal to 1 , it can be summarized as follows (see [9] for a translation in terms of exponents of Diophantine approximation). Dirichlet's box principle yields (under the assumption that $\xi_{0}, \ldots, \xi_{r}$ are linearly independent over $\mathbb{Q}$ ) very good simultaneous approximants $p_{1} / p_{0}, \ldots, p_{r} / p_{0}$ to $\xi_{1} / \xi_{0}, \ldots, \xi_{r} / \xi_{0}$ with the same denominator $p_{0}$. This means that $\left(\xi_{0}, \ldots, \xi_{r}\right)$ is suffi- 
ciently close to the line generated by $\left(p_{0}, \ldots, p_{r}\right)$ in $\mathbb{R}^{r+1}$, and contradicts (if $r<\tau$ ) the lower bound proved in the first induction step of Nesterenko's proof (see above). Actually, this first step is very easy to prove directly (without Nesterenko's machinery for controlling the intersection of a linear subspace with a hyperplane). Indeed, for some $n$ (denoted by $k_{n}$ in Sect. 2.1) the hyperplane $H_{n}$ defined by $\ell_{0, n} X_{0}+\cdots+$ $\ell_{r, n} X_{r}=0$ has comparatively small height and is very close to $\left(\xi_{0}, \ldots, \xi_{r}\right)$, hence to $\left(p_{0}, \ldots, p_{r}\right)$, so that $\left(p_{0}, \ldots, p_{r}\right)$ has to belong to $H_{n}$. But then the distance from $\left(\xi_{0}, \ldots, \xi_{r}\right)$ to $H_{n}$ is less than, or equal to, the distance of $\left(\xi_{0}, \ldots, \xi_{r}\right)$ to $\left(p_{0}, \ldots, p_{r}\right)$; this is too small, in contradiction with the lower bound for $\left|L_{n}(\xi)\right|$.

At last, let us comment briefly on the optimality of our criterion. Eventhough Assumptions (i) and (ii) might be refined (see above), it is likely that the conclusion $s \geq \tau+\gamma_{1}+\cdots+\gamma_{s}$ of Theorem 1 cannot be improved (see [9,8] for related results when $s=1$ ). Now it turns out that Theorem 1 applied to the construction of $[2,16]$ gives

$$
\operatorname{dim}_{\mathbb{Q}} \operatorname{Span}_{\mathbb{Q}}(1, \zeta(3), \zeta(5), \zeta(7), \ldots, \zeta(a)) \geq \frac{1+o(1)}{1+\log 2} \log a,
$$

exactly like Nesterenko's linear independence criterion, because $\gamma_{1}, \ldots, \gamma_{s}$ turn out to be very small (and the improvement lies inside the error term). Therefore a new construction of linear forms seems to be necessary for refining this lower bound.

\section{Applications of the criterion}

\subsection{First application: odd zeta values}

For a pair of positive integers $s$ and $t$ with $t<s$, consider the (very-well-poised) hypergeometric series

$$
h_{n}=2 n !^{2(s-t)} \sum_{k=1}^{\infty}\left(k+\frac{n}{2}\right) \frac{\prod_{j=1}^{t n}(k-j) \cdot \prod_{j=1}^{t n}(k+n+j)}{\prod_{j=0}^{n}(k+j)^{2 s}} .
$$

It is known $[2,16,20]$ that, for some $a_{i, n} \in \mathbb{Q}$,

$$
h_{n}=a_{0, n}+\sum_{i=1}^{s-1} a_{i, n} \zeta(2 i+1)
$$

Let us summarize the auxiliary results about these linear forms that can be deduced from $[11,20]$.

Denote by $x_{0}$ the maximal real zero of the polynomial

$$
\left(x+t+\frac{1}{2}\right)\left(x-\frac{1}{2}\right)^{2 s+1}-\left(x-t-\frac{1}{2}\right)\left(x+\frac{1}{2}\right)^{2 s+1} ;
$$


it belongs to the interval $\left(t+\frac{1}{2},+\infty\right)$. Introduce the function

$$
\begin{aligned}
f(x)= & \left(t+\frac{1}{2}\right) \log \left(x+t+\frac{1}{2}\right)+\left(t+\frac{1}{2}\right) \log \left(x-t-\frac{1}{2}\right) \\
& -\left(s+\frac{1}{2}\right) \log \left(x+\frac{1}{2}\right)-\left(s+\frac{1}{2}\right) \log \left(x-\frac{1}{2}\right) .
\end{aligned}
$$

Consider the following product over primes:

$$
\widehat{\Pi}_{n}=\widehat{\Pi}_{n}^{(t)}=\prod_{l=2}^{2 t-1} \prod_{\substack{(t+1) n \\ \text { an } \\\{n\} \in E_{l}}} p^{l-1},
$$

where

$$
\begin{aligned}
E_{2 l} & =\left[\frac{l}{t}, \frac{l+1}{t+1 / 2}\right) \quad \text { for } l=0,1, \ldots, t-1, \\
E_{2 l-1} & =\left[\frac{l}{t+1 / 2}, \frac{l}{t}\right) \quad \text { for } l=1,2, \ldots, t,
\end{aligned}
$$

and $\{\cdot\}$ denotes the fractional part of a number.

Proposition 2 In the above notation,

$$
\lim _{n \rightarrow \infty} \frac{\log \left|h_{n}\right|}{n}=f\left(x_{0}\right)
$$

and

$$
\begin{aligned}
\limsup _{n \rightarrow \infty} \frac{\log \left|a_{i, n}\right|}{n} \leq & \operatorname{Re} f(0)=2(s-t) \log 2 \\
& +(2 t+1) \log (2 t+1) \text { for } i=0,1, \ldots, s-1 .
\end{aligned}
$$

Moreover, the rational coefficients of the forms (5) satisfy

$$
d_{n}^{2 s-1} \widehat{\Pi}_{n}^{-1} a_{0, n} \in \mathbb{Z} \quad \text { and } \quad d_{n}^{2(s-i)-2} \widehat{\Pi}_{n}^{-1} a_{i, n} \in \mathbb{Z} \text { for } i=1, \ldots, s-1,
$$

while the asymptotic behavior of (8) is determined by

$$
\lim _{n \rightarrow \infty} \frac{\log \widehat{\Pi}_{n}}{n}=(2 t-1) \psi(2)-\sum_{l=1}^{t} \psi\left(1+\frac{l}{t}\right)-\sum_{l=2}^{t} \psi\left(1+\frac{l}{t+1 / 2}\right)
$$

where $\psi(x)$ is the digamma function (that is, the logarithmic derivative of the Gamma function). 
Proof of Proposition 2 This result follows from Propositions 2.1, 3.1, and 4.1 and Lemma 4.5 of [20], taking also into account the proof [11] of the so-called 'denominator conjecture' by Krattenthaler and Rivoal. They show that

$$
d_{n}^{2 s-1} a_{0, n} \in \mathbb{Z} \quad \text { and } \quad d_{n}^{2(s-i)-2} a_{i, n} \in \mathbb{Z} \text { for } i=1, \ldots, s-1,
$$

in other words, they get rid of an extra $d_{n}$ with respect to the analogous properties used in [20].

Proof of Theorem 3 The collection of numbers under consideration is

$$
\left(\xi_{0}, \xi_{1}, \xi_{2}, \ldots, \xi_{s-1}\right)=(1, \zeta(3), \zeta(5), \ldots, \zeta(2 s-1))
$$

Setting

$$
\ell_{i, n}=d_{n}^{2 s-1} \widehat{\Pi}_{n}^{-1} a_{i, n} \in \mathbb{Z} \text { for } i=0, \ldots, s-1,
$$

from (10) we see that

$$
d_{n}^{2 i+1} \mid \ell_{i, n} \text { for } i=1, \ldots, s-1,
$$

hence in the notation of Corollary 1 we have $r=s-1, \delta_{i, n}=d_{n}^{2 i+1}$,

$$
\log \alpha=f\left(x_{0}\right)+2 s-1-\widehat{\varpi}_{t}
$$

and

$$
\log \beta=2(s-t) \log 2+(2 t+1) \log (2 t+1)+2 s-1-\widehat{\varpi}_{t},
$$

where $\widehat{\omega}_{t}=\lim _{n \rightarrow \infty} \frac{\log \widehat{\Pi}_{n}}{n}$.

Using standard formulas for the digamma function we can write $\widehat{\varpi}_{t}$ by means of elementary functions only:

$$
\begin{aligned}
\widehat{\varpi}_{t}= & 3 t-\frac{1}{2}-\left(2 t+\frac{1}{2}\right) \sum_{l=1}^{t} \frac{1}{l}+\frac{\pi}{2} \sum_{l=2}^{t} \cot \frac{2 \pi l}{2 t+1}+2 \sum_{l=1}^{t} \cos \frac{4 \pi l}{2 t+1} \log \sin \frac{\pi l}{2 t+1} \\
& -\log 2+t \log t+\left(t-\frac{1}{2}\right) \log (2 t+1) .
\end{aligned}
$$

We now apply Corollary 1 . With the choice $s=70, t=10$ we obtain

$$
1-\frac{\log \alpha-3}{\log \beta}=2.0004232415 \ldots>2
$$

hence

$$
\operatorname{dim}_{\mathbb{Q}} \operatorname{Span}_{\mathbb{Q}}(1, \zeta(3), \zeta(5), \ldots, \zeta(139)) \geq 3
$$


in the same way, taking $s=981, t=65$ we get

$$
1-\frac{\log \alpha-(3+5)}{\log \beta}=3.0003426048 \ldots>3
$$

yielding

$$
\operatorname{dim}_{\mathbb{Q}} \operatorname{Span}_{\mathbb{Q}}(1, \zeta(3), \zeta(5), \ldots, \zeta(1961)) \geq 4
$$

This computation implies Theorem 3.

\subsection{Second application: odd $q$-zeta values}

We now fix a number $q$ of the form $1 / p$, where $p \in \mathbb{Z} \backslash\{0, \pm 1\}$. As in the previous section, we take a pair of positive integers $s$ and $t$ satisfying $t<s$. With the help of the basic hypergeometric series

$$
\begin{aligned}
h_{n}(q) & =(q)_{n}^{2(s-t)} \sum_{k=1}^{\infty}\left(1-q^{2 k+n}\right) \frac{\left(q^{k-t n}\right)_{t n} \cdot\left(q^{k+n+1}\right)_{t n}}{\left(q^{k}\right)_{n+1}^{2 s}} q^{k(s-t) n+k s-k} \\
& =a_{0, n}(q)+\sum_{i=1}^{s-1} a_{i, n}(q) \zeta_{q}(2 i+1)
\end{aligned}
$$

where $(b)_{n}=(b ; q)_{n}=\prod_{k=1}^{n}\left(1-q^{k-1} b\right)$ is the $q$-Pochhammer symbol, it was shown in [12] (see also [10], where the ' $q$-denominator conjecture' is proved) that

$$
\operatorname{dim}_{\mathbb{Q}} \operatorname{Span}_{\mathbb{Q}}\left(1, \zeta_{q}(3), \zeta_{q}(5), \ldots, \zeta_{q}(2 s-1)\right) \geq \frac{\pi+o(1)}{2 \sqrt{\pi^{2}+12}} \sqrt{2 s}
$$

as $s \rightarrow \infty$. The coefficients $a_{i, n}(q)$ are, in fact, rational functions of the variable $p=1 / q$, whose denominators involve only powers of $p$ and of the cyclotomic polynomials

$$
\Phi_{j}(p)=\prod_{\substack{k=1 \\(k, j)=1}}^{j}\left(p-e^{2 \pi \sqrt{-1} k / j}\right) \in \mathbb{Z}[p], \quad \operatorname{deg}_{p} \Phi_{j}(p)=\varphi(j), \quad j=1,2, \ldots
$$

In these settings, the $q$-analog of the quantity $d_{n}$ is the least common multiple of the polynomials $p-1, p^{2}-1, \ldots, p^{n}-1$, which equals

$$
d_{n}(p)=\prod_{j=1}^{n} \Phi_{j}(p)
$$


Mertens' theorem asserts that, for a real number $p$ with $|p|>1$,

$$
\lim _{n \rightarrow \infty} \frac{\log \left|d_{n}(p)\right|}{n^{2} \log |p|}=\frac{3}{\pi^{2}} .
$$

The following statement summarizes the analytic and arithmetic results of $[10,12]$ for the linear forms in the odd $q$-zeta values, sharpened using the argument of [20, Sect. 4]: one just replaces primes by cyclotomic polynomials (12) (cf. [21, Sect. 1]). Recall that the sets $E_{l}$ are defined in (9).

Proposition 3 In the above notation,

$$
\lim _{n \rightarrow \infty} \frac{\log \left|h_{n}(q)\right|}{n^{2} \log |p|}=-t(s-t),
$$

and

$$
\limsup _{n \rightarrow \infty} \frac{\log \left|a_{i, n}(q)\right|}{n^{2} \log |p|} \leq \frac{s+2 t^{2}}{4} \text { for } i=0,1, \ldots, s-1 .
$$

Moreover, the coefficients of the forms (11) satisfy

$$
\begin{aligned}
& (2 s) ! p^{M} d_{n}(p)^{2 s-1} \widehat{\Pi}_{n}(p)^{-1} a_{0, n}(q) \in \mathbb{Z}[p] \quad \text { and } \\
& (2 s) ! p^{M} d_{n}(p)^{2(s-i)-2} \widehat{\Pi}_{n}(p)^{-1} a_{i, n}(q) \in \mathbb{Z}[p] \text { for } i=1, \ldots, s-1,
\end{aligned}
$$

where

$$
\begin{aligned}
M & =\left\lceil\frac{s(n+1)^{2}}{4}\right\rceil+\frac{t n(t n-1)}{2}+(2 s+1) n-\left\lfloor\frac{(s-t) n}{2}\right\rfloor, \\
\widehat{\Pi}_{n}(p) & =\widehat{\Pi}_{n}^{(t)}(p)=\prod_{l=2}^{2 t-1} \prod_{\substack{(t+1) n<j \leq n \\
\{n / j\} \in E_{l}}} \Phi_{j}(p)^{l-1},
\end{aligned}
$$

and the asymptotic behavior of (14) is determined by

$$
\begin{aligned}
\lim _{n \rightarrow \infty} \frac{\log \widehat{\Pi}_{n}(p)}{n^{2} \log |p|} & =\widehat{\omega}_{t}^{\prime}=(2 t-1) \psi_{1}(2)-\sum_{l=1}^{t} \psi_{1}\left(1+\frac{l}{t}\right)-\sum_{l=2}^{t} \psi_{1}\left(1+\frac{l}{t+1 / 2}\right) \\
& =\frac{(t-1)^{2}}{2}+\frac{3}{\pi^{2}}\left(2 t-1-t^{2} \sum_{l=1}^{t} \frac{1}{l^{2}}\right)-\sum_{l=2}^{t} \psi_{1}\left(1+\frac{l}{t+1 / 2}\right),
\end{aligned}
$$

where

$$
\psi_{1}(x)=\frac{3}{\pi^{2}} \frac{\mathrm{d} \psi(x)}{\mathrm{d} x}=-\frac{3}{\pi^{2}} \sum_{k=0}^{\infty} \frac{1}{(x+k)^{2}}
$$

denotes the (normalized) trigamma function. 
Proof of Theorem 4 In the notation $p=1 / q \in \mathbb{Z} \backslash\{0, \pm 1\}$, set

$$
\ell_{i, n}=p^{M} d_{n}(p)^{2 s-1} \widehat{\Pi}_{n}(p)^{-1} a_{i, n}(q) \in \mathbb{Z} \text { for } i=0, \ldots, s-1 .
$$

To these linear forms in

$$
\left(\xi_{0}, \xi_{1}, \xi_{2}, \ldots, \xi_{s-1}\right)=\left(1, \zeta_{q}(3), \zeta_{q}(5), \ldots, \zeta_{q}(2 s-1)\right)
$$

we apply Theorem 1 taking $Q_{n}=\beta^{n^{2} \log |p|}$ and $\tau=-(\log \alpha) /(\log \beta)$, where

$$
\log \alpha=-t(s-t)+\frac{s+2 t^{2}}{4}+\frac{3(2 s-1)}{\pi^{2}}-\widehat{\varpi}_{t}^{\prime}
$$

and

$$
\log \beta=\frac{s+2 t^{2}}{2}+\frac{3(2 s-1)}{\pi^{2}}-\widehat{\varpi}_{t}^{\prime}
$$

From (13) we see that

$$
d_{n}(p)^{2 i+1} \mid \ell_{i, n} \text { for } i=1, \ldots, s-1
$$

hence we may take $\delta_{i, n}=d_{n}(p)^{2 i+1}$ to meet the required conditions of Theorem 1 . The existence of an odd integer $3 \leq i_{0} \leq 9$, for which $\zeta_{q}\left(i_{0}\right)$ is irrational, is already shown in [10]. The following choices of $s$ and $t$ and Theorem 1 ensure the truth of Theorem 4:

$$
\begin{array}{ll}
s=19, \quad t=4: \quad 1-\frac{\log \alpha-3 \cdot 3 / \pi^{2}}{\log \beta}=2.0300573456 \ldots>2, \\
s=42, \quad t=6: \quad 1-\frac{\log \alpha-(3+5) \cdot 3 / \pi^{2}}{\log \beta}=3.0344397971 \ldots>3, \\
s=73, \quad t=8: \quad 1-\frac{\log \alpha-(3+5+7) \cdot 3 / \pi^{2}}{\log \beta}=4.0108485236 \ldots>4 .
\end{array}
$$

\subsection{Third application: $\log 2$ and odd zeta values}

As in the two previous sections, we take a pair of positive integers $s$ and $t$ with $t<s$, but this time we assume $s$ to be even. Consider the hypergeometric series

$$
\widetilde{h}_{n}=2 \frac{\left(2^{-2 n}(2 n) !\right)^{s}}{n !^{2 t}} \sum_{k=1}^{\infty}\left(k+\frac{n}{2}\right) \frac{\prod_{j=1}^{t n}(k-j) \cdot \prod_{j=1}^{t n}(k+n+j)}{\prod_{j=0}^{2 n}(k+j / 2)^{s}}
$$


Its $k$-rational summand

$$
H_{n}(k)=(2 k+n) \frac{\prod_{j=1}^{t n}(k-j)}{n !^{t}} \frac{\prod_{j=1}^{t n}(k+n+j)}{n !^{t}}\left(\frac{2^{-2 n}(2 n) !}{\prod_{j=0}^{2 n}(k+j / 2)}\right)^{s}
$$

differs from the corresponding one in (4) a little: the $s$ products $\left(n ! / \prod_{j=0}^{n}(k+j)\right)^{2}$ in (4) are replaced by $2^{-2 n}(2 n) ! / \prod_{j=0}^{2 n}(k+j / 2)$ in (15), and these two have similar asymptotics as $n \rightarrow \infty$. This similarity allows us to compute, like in [2] or [20], the asymptotic behavior of (15) and of the coefficients in the 'zeta' decomposition of (15) which we are going to describe in the next statement.

Lemma 1 In the above notation, we have

$$
\widetilde{h}_{n}=\widetilde{a}_{0, n}+\widetilde{a}_{1, n} \log 2+\sum_{i=2}^{s / 2} \widetilde{a}_{i, n} \zeta(2 i-1)
$$

where

$$
2^{4 t n} d_{2 n}^{s} \widetilde{a}_{0, n} \in \mathbb{Z} \text { and } 2^{4 t n} d_{2 n}^{s-2 i+1} \widetilde{a}_{i, n} \in \mathbb{Z} \text { for } i=1,2, \ldots, s / 2
$$

Proof The function (16) is the product of integer-valued polynomials $2 k+n$,

$$
\frac{\prod_{j=1}^{n}(k-l n-j)}{n !}, \quad l=0,1, \ldots, t-1, \quad \text { and } \quad \frac{\prod_{j=1}^{n}(k+l n+j)}{n !}, \quad l=1,2, \ldots, t
$$

and of $s$ copies of the rational function

$$
\frac{2^{-2 n}(2 n) !}{\prod_{j=0}^{2 n}(k+j / 2)}=\frac{2 \cdot(2 n) !}{\prod_{j=0}(2 k+j)}=\sum_{j=0}^{2 n} \frac{(-1)^{j}\left(\begin{array}{c}
2 n \\
j
\end{array}\right)}{k+j / 2}
$$

It follows from the Leibniz rule for differentiating a product (cf. [20, Lemmas 1.2-1.4] and the formula (29)) that

$$
H_{n}(k)=\sum_{i=1}^{s} \sum_{j=0}^{2 n} \frac{A_{i, j}}{(k+j / 2)^{i}}
$$

with the property

$$
2^{4 t n} d_{2 n}^{s-i} A_{i, k} \in \mathbb{Z} .
$$


For a variable $x$ in the unit circle $|x|<1$, we now perform the summation

$$
\begin{aligned}
\sum_{k=1}^{\infty} & H_{n}(k) x^{2 k}=\sum_{k=1}^{\infty} x^{2 k} \sum_{i=1}^{s} \sum_{j=0}^{2 n} \frac{A_{i, j}}{(k+j / 2)^{i}}=\sum_{i=1}^{s} \sum_{j=0}^{2 n} A_{i, j} x^{-j} \sum_{k=1}^{\infty} \frac{x^{2 k+j}}{(k+j / 2)^{i}} \\
= & \sum_{i=1}^{s} \sum_{j=0}^{n} A_{i, 2 j} x^{-2 j}\left(\sum_{k=1}^{\infty}-\sum_{k=1}^{j}\right) \frac{x^{2 k}}{k^{i}} \\
& +\sum_{i=1}^{s} \sum_{j=1}^{n} A_{i, 2 j-1} x^{-2 j+1}\left(\sum_{k=1}^{\infty}-\sum_{k=1}^{j}\right) \frac{x^{2 k-1}}{(k-1 / 2)^{i}} \\
= & \sum_{i=1}^{s} \operatorname{Li}_{i}\left(x^{2}\right) \sum_{j=0}^{n} A_{i, 2 j} x^{-2 j}+\sum_{i=1}^{s}\left(2^{i} \operatorname{Li}_{i}(x)-\operatorname{Li}_{i}\left(x^{2}\right)\right) \sum_{j=1}^{n} A_{i, 2 j-1} x^{-2 j+1} \\
& -\sum_{i=1}^{s} \sum_{j=0}^{n} A_{i, 2 j} \sum_{k=1}^{j} \frac{x^{2 k-2 j}}{k^{i}}-\sum_{i=1}^{s} \sum_{j=1}^{n} A_{i, 2 j-1} \sum_{k=1}^{j} \frac{x^{2 k-2 j}}{(k-1 / 2)^{i}}
\end{aligned}
$$

where

$$
\operatorname{Li}_{i}(x)=\sum_{k=1}^{\infty} \frac{x^{k}}{k^{i}}
$$

denotes the $i$ th polylogarithm function. To compute the limit $x \rightarrow 1^{-}$in (21), we use Abel's theorem for power series, the sum residue theorem in the form

$$
\sum_{j=0}^{n} A_{1,2 j}+\sum_{j=1}^{n} A_{1,2 j-1}=\sum_{j=0}^{2 n} \operatorname{Res}_{k=-j / 2} H_{n}(k)=-\operatorname{Res}_{k=\infty} H_{n}(k)=0
$$

and the identity $\operatorname{Li}_{1}(x)-\operatorname{Li}_{1}\left(x^{2}\right)=-\operatorname{Li}_{1}(-x)$. Therefore,

$$
\begin{aligned}
\widetilde{h}_{n}= & \log 2 \cdot 2 \sum_{j=1}^{n} A_{1,2 j-1}+\sum_{i=2}^{s} \zeta(i)\left(\sum_{j=0}^{n} A_{i, 2 j}+\left(2^{i}-1\right) \sum_{j=1}^{n} A_{i, 2 j-1}\right) \\
& -\sum_{i=1}^{s}\left(\sum_{j=0}^{n} A_{i, 2 j} \sum_{k=1}^{j} \frac{1}{k^{i}}+\sum_{j=1}^{n} A_{i, 2 j-1} \sum_{k=1}^{j} \frac{1}{(k-1 / 2)^{i}}\right),
\end{aligned}
$$

where we used the evaluations $-\operatorname{Li}_{1}(-1)=\log 2$ and $\operatorname{Li}_{i}(1)=\zeta(i)$ for $i=2, \ldots, s$. Finally, note that the parity of $s$ implies from (16) that

$$
H_{n}(-k-n)=-H_{n}(k),
$$


hence $A_{i, j}=(-1)^{i-1} A_{i, 2 n-j}$ and $\sum_{j=0}^{n} A_{i, 2 j}=\sum_{j=1}^{n} A_{i, 2 j-1}=0$ for $i=$ $2,4, \ldots, s$. This implies the required decomposition (17) with

$$
\begin{aligned}
& \widetilde{a}_{0, n}=-\sum_{i=1}^{s}\left(\sum_{j=0}^{n} A_{i, 2 j} \sum_{k=1}^{j} \frac{1}{k^{i}}+\sum_{j=1}^{n} A_{i, 2 j-1} \sum_{k=1}^{j} \frac{1}{(k-1 / 2)^{i}}\right), \\
& \widetilde{a}_{1, n}=2 \sum_{j=1}^{n} A_{1,2 j-1}, \quad \text { and } \\
& \widetilde{a}_{i, n}=\sum_{j=0}^{n} A_{2 i-1,2 j}+\left(2^{2 i-1}-1\right) \sum_{j=1}^{n} A_{2 i-1,2 j-1} \quad \text { for } i=2, \ldots, s / 2 .
\end{aligned}
$$

Using (20) we arrive at the inclusions (18).

We are now in power to sharpen the inclusions (18) in the way we already did in Propositions 2 and 3. Note that for an integer $N>2$ and a prime $p>\sqrt{2 N}$ we have $\operatorname{ord}_{p} \Gamma(N+1)=\operatorname{ord}_{p} N !=\left\lfloor\frac{N}{p}\right\rfloor \quad$ and $\quad \operatorname{ord}_{p} \frac{\Gamma(N+1 / 2)}{\Gamma(1 / 2)}=\operatorname{ord}_{p} \frac{(2 N) !}{2^{2 N} N !}=\left\lfloor\frac{N}{p}\right\rfloor$,

where

$$
\llbracket x \Perp=\lfloor 2 x\rfloor-\lfloor x\rfloor
$$

(see the proof of Lemma 3 for another expression of $\llbracket x \Downarrow$ ).

Lemma 2 For the coefficients in the decomposition (17), we have the inclusions

$$
2^{4 t n} d_{2 n}^{s} \widetilde{\Pi}_{n}^{-1} \widetilde{a}_{0, n} \in \mathbb{Z} \text { and } 2^{4 t n} d_{2 n}^{s-2 i+1} \widetilde{\Pi}_{n}^{-1} \widetilde{a}_{i, n} \in \mathbb{Z} \text { for } i=1,2, \ldots, s / 2,
$$

where

$$
\widetilde{\Pi}_{n}=\widetilde{\Pi}_{n}^{(t)}=\prod_{\sqrt{2(t+1) n}<p \leq 2 n} p^{\tau(n / p)}
$$

and the function $\tau(\cdot)$ is defined as follows:

$$
\begin{aligned}
\tau(x) & =\tau_{t}(x)=\min _{y \in \mathbb{R}}\left\{\tau_{1}(x, y), \tau_{2}(x, y)\right\}, \\
\tau_{1}(x, y) & =\left\lfloor\left(t+\frac{1}{2}\right) x+\frac{y}{2}\right\rfloor+\left\lfloor\left(t+\frac{1}{2}\right) x-\frac{y}{2}\right\rfloor-\left\lfloor\frac{x}{2}+\frac{y}{2}\right\rfloor-\left\lfloor\frac{x}{2}-\frac{y}{2}\right\rfloor-2 t\lfloor x\rfloor, \\
\tau_{2}(x, y) & =\left\lfloor\left(t+\frac{1}{2}\right) x+\frac{y}{2}\right\rfloor+\left\lfloor\left(t+\frac{1}{2}\right) x-\frac{y}{2}\right\rfloor-\left\lfloor\frac{x}{2}+\frac{y}{2}\right\rfloor-\left\lfloor\frac{x}{2}-\frac{y}{2}\right\rfloor-2 t\lfloor x\rfloor .
\end{aligned}
$$


Proof In the notation of the above proof of Lemma 1, we can write

$$
\begin{aligned}
A_{i, j}= & \left.\frac{1}{(s-i) !} \frac{\mathrm{d}^{s-i}}{\mathrm{~d} k^{s-i}}\left(H_{n}(k)\left(k+\frac{j}{2}\right)^{s}\right)\right|_{k=-j / 2} \\
& \text { for } i=1, \ldots, s \text { and } j=0,1, \ldots, 2 n .
\end{aligned}
$$

Taking into account the partial fraction decomposition (19) and

$$
\begin{aligned}
& \left.\frac{\prod_{l=1}^{t n}(k-l)}{n !^{t}} \frac{\prod_{l=1}^{t n}(k+n+l)}{n !^{t}}\right|_{k=-j / 2} \\
& =(-1)^{t n} \frac{\Gamma((2 t+1) n / 2+(j-n) / 2+1)}{n !^{t} \Gamma(n / 2+(j-n) / 2+1)} \frac{\Gamma((2 t+1) n / 2+(n-j) / 2+1)}{n !^{t} \Gamma(n / 2+(n-j) / 2+1)} \\
& \quad \text { for } j=0,1, \ldots, 2 n,
\end{aligned}
$$

with the help of [20, Lemma 4.1] we conclude that any common multiple $\Pi$ of the numbers in (30), involving primes $p \leq 2 n$ only, can be used in sharpening the inclusions (20):

$$
2^{2 t n} d_{2 n}^{s-i} \Pi^{-1} A_{i, j} \in \mathbb{Z} \text { for } i=1, \ldots, s \quad \text { and } \quad j=0,1, \ldots, 2 n
$$

In view of (23), it is enough to show that $\widetilde{\Pi}_{n}$ defined in (27) is such a multiple. From (24) we see that

$$
\begin{gathered}
\operatorname{ord}_{p} \frac{\Gamma((2 t+1) n / 2+(j-n) / 2+1)}{n !^{t} \Gamma(n / 2+(j-n) / 2+1)} \frac{\Gamma((2 t+1) n / 2+(n-j) / 2+1)}{n !^{t} \Gamma(n / 2+(n-j) / 2+1)} \\
\quad=\left\{\begin{array}{ll}
\tau_{1}(n / p,(j-n) / p) & \text { for } j \text { even, } \\
\tau_{2}(n / p,(j-n) / p) & \text { for } j \text { odd, }
\end{array} \quad j=0,1, \ldots, 2 n,\right.
\end{gathered}
$$

and this implies the desired result.

Lemma 3 The quantity (27) can be written as follows:

$$
\widetilde{\Pi}_{n}=\widetilde{\Pi}_{n}^{(t)}=\prod_{l=1}^{2 t-1} \prod_{\substack{\sqrt{2(t+1) n}<p \leq 2 n \\\{n / p\} \in E_{l}}} p^{l}
$$

where the sets $E_{l}$ are given in (9). In addition,

$$
\begin{aligned}
& \lim _{n \rightarrow \infty} \frac{\log \widetilde{\Pi}_{n}}{n}=\widetilde{\varpi}_{t}=4\left\lfloor\frac{t}{2}\right\rfloor-\left(2 t+\frac{1}{2}\right) \sum_{l=1}^{\lfloor t / 2\rfloor} \frac{1}{l}+\frac{\pi}{2} \sum_{l=1}^{t} \cot \frac{2 \pi l}{2 t+1} \\
& +t \log t+\left(t+\frac{1}{2}\right) \log (2 t+1) \text {. }
\end{aligned}
$$


Proof Using the identity $\lfloor 2 x\rfloor=\lfloor x\rfloor+\lfloor x+1 / 2\rfloor$, we see that the function (25) is equal to $\lfloor x+1 / 2\rfloor$. This implies that $\tau_{2}(x, y)=\tau_{1}(x, y+1)$, hence

$$
\tau(x)=\min _{y \in \mathbb{R}}\left\{\tau_{1}(x, y)\right\} .
$$

Furthermore, it follows from (28) that $\tau_{1}(x+1, y)=\tau_{1}(x, y+1)$, hence the function $\tau(x)$ is 1-periodic:

$$
\tau(x)=\tau(\{x\})
$$

Moreover, we have $\tau_{1}(x, y+2)=\tau_{1}(x, y)$ and $\tau_{1}(x,-y)=\tau_{1}(x, y)$ implying that the minimum in (33) can be performed for $y \in[0,1[$ only:

$$
\tau(x)=\min _{0 \leq y<1}\left\{\tau_{1}(x, y)\right\} .
$$

It remains to use the results of [20, Section 4] (already taken into account in Sects. 3.1 and 3.2):

$$
\min _{0 \leq y<1}\left\{\tau_{1}(x, y)\right\}=l \text { for } x \in E_{l}, \quad l=0,1, \ldots, 2 t-1,
$$

where the sets $E_{0}, E_{1}, \ldots, E_{2 t-1}$ are defined in (9); this gives us the desired form (31) of the quantity (27).

To compute the asymptotics in (32) we apply [20, Lemma 4.4]:

$$
\begin{aligned}
& \lim _{n \rightarrow \infty} \frac{\log \widetilde{\Pi}_{n}}{n}=\sum_{l=1}^{2 t-1} l\left(\int_{E_{l} \cap[0,1 / 2)} \mathrm{d} \psi(1+x)+\int_{E_{l} \cap[1 / 2,1)} \mathrm{d} \psi(x)\right) \\
& =\sum_{l=1}^{2 t-1} l\left(\int_{E_{l}} \mathrm{~d} \psi(1+x)+\int_{E_{l} \cap[1 / 2,1)} \mathrm{d}\left(-\frac{1}{x}\right)\right) \\
& =\varpi_{t}+\sum_{l=\lfloor t / 2\rfloor}^{t-1} 2 l \int_{E_{2 l}} \mathrm{~d}\left(-\frac{1}{x}\right)-2\left\lfloor\frac{t}{2}\right\rfloor \int_{\lfloor t / 2\rfloor / t}^{1 / 2} \mathrm{~d}\left(-\frac{1}{x}\right) \\
& +\sum_{l=\lfloor t / 2\rfloor+1}^{t}(2 l-1) \int_{E_{2 l-1}} \mathrm{~d}\left(-\frac{1}{x}\right) \\
& =\varpi_{t}+\sum_{l=\lfloor t / 2\rfloor}^{t-1} 2 l\left(\frac{t}{l}-\frac{t+1 / 2}{l+1}\right)-2\left\lfloor\frac{t}{2}\right\rfloor\left(\frac{t}{\lfloor t / 2\rfloor}-2\right) \\
& +\sum_{l=\lfloor t / 2\rfloor+1}^{t}(2 l-1)\left(\frac{t+1 / 2}{l}-\frac{t}{l}\right) \\
& =\varpi_{t}+\left(2 t+\frac{1}{2}\right) \sum_{l=\lfloor t / 2\rfloor+1}^{t} \frac{1}{l}-2\left(t-2\left\lfloor\frac{t}{2}\right\rfloor\right),
\end{aligned}
$$


where $\varpi_{t}$ is defined by

$$
\begin{aligned}
\varpi_{t} & =2 t(1-\gamma)-\left(2 t+\frac{1}{2}\right) \sum_{l=1}^{t} \frac{1}{l}-\sum_{l=1}^{t}\left(\psi\left(\frac{l}{t}\right)+\psi\left(\frac{l}{t+1 / 2}\right)\right) \\
& =2 t \psi(2)-\sum_{l=1}^{t}\left(\psi\left(1+\frac{l}{t}\right)+\psi\left(1+\frac{l}{t+1 / 2}\right)\right),
\end{aligned}
$$

and $\gamma=-\psi(1)$ is Euler's constant. It remains to apply identities for the digamma function, and the lemma follows.

The following statement summarizes our findings in this section.

Proposition 4 For positive integers $s$ and $t$ with $s$ even and $t<s$, the linear forms (15), (17) and their coefficients admit the asymptotics

$$
\lim _{n \rightarrow \infty} \frac{\log \left|\widetilde{h}_{n}\right|}{n}=f\left(x_{0}\right)
$$

and

$\limsup _{n \rightarrow \infty} \frac{\log \left|\widetilde{a}_{i, n}\right|}{n} \leq \operatorname{Re} f(0)=2(s-t) \log 2+(2 t+1) \log (2 t+1)$ for $i=0,1, \ldots, s / 2$,

where $x_{0} \in\left(t+\frac{1}{2},+\infty\right)$ is the maximal real zero of the polynomial (6) and the function $f(x)$ is defined in (7). Moreover, the coefficients in the decomposition (17) satisfy (26) and the asymptotics of the quantities (27), (31) is determined in (32).

Proof of Theorem 5 This time we have $\mathbb{Q}$-linear forms in

$$
\left(\xi_{0}, \xi_{1}, \xi_{2}, \ldots, \xi_{s / 2}\right)=(1, \log 2, \zeta(3), \zeta(5), \ldots, \zeta(s-1))
$$

whose coefficients are

$$
\ell_{i, n}=2^{4 t n} d_{2 n}^{s} \widetilde{\Pi}_{n}^{-1} \widetilde{a}_{i, n} \in \mathbb{Z} \text { for } i=0,1, \ldots, s / 2
$$

Then (26) implies

$$
d_{2 n}^{2 i-1} \mid \ell_{i, n} \text { for } i=1,2, \ldots, s / 2 \text {, }
$$

hence in the notation of Corollary 1 we have $r=s / 2, \delta_{i, n}=d_{2 n}^{2 i-1}$,

$$
\log \alpha=f\left(x_{0}\right)+4 t \log 2+2 s-\widetilde{\varpi}_{t}
$$

and

$$
\log \beta=2(s+t) \log 2+(2 t+1) \log (2 t+1)+2 s-\widetilde{\varpi}_{t} .
$$


Applying the theorem with the choice $s=94, t=11$ we obtain

$$
1-\frac{\log \alpha-2}{\log \beta}=2.0064440535 \ldots>2 \text {, }
$$

while the choice $s=1152, t=67$ results in

$$
1-\frac{\log \alpha-(2+6)}{\log \beta}=3.0004493689 \ldots>3
$$

This implies the required independence result.

Remark 3 Applying Nesterenko's linear independence criterion instead of Corollary 1 yields the same bound on $i_{1}$, and the slightly less precise bound $i_{2} \leq 1155$.

\subsection{Triple integrals for rational approximations to $\log 2$}

The particular case $s=2, t=1$ of our construction in Sect. 3.3 is of independent interest, since the corresponding series

$$
\widetilde{h}_{n}=2\left(\frac{2^{-2 n}(2 n) !}{n !}\right)^{2} \sum_{k=1}^{\infty}\left(k+\frac{n}{2}\right) \frac{\prod_{j=1}^{n}(k-j) \cdot \prod_{j=1}^{n}(k+n+j)}{\prod_{j=0}^{2 n}(k+j / 2)^{2}}
$$

goes in parallel with Ball's series

$$
h_{n}=2 n !^{2} \sum_{k=1}^{\infty}\left(k+\frac{n}{2}\right) \frac{\prod_{j=1}^{n}(k-j) \cdot \prod_{j=1}^{n}(k+n+j)}{\prod_{j=0}^{n}(k+j)^{4}}
$$

for Apéry's approximations to $\zeta(3)$. Switching to the classical hypergeometric notation [17] and using

$$
\frac{2^{-2 n}(2 n) !}{n !}=\frac{\Gamma\left(n+\frac{1}{2}\right)}{\Gamma\left(\frac{1}{2}\right)}=\frac{\Gamma(n+1 / 2)}{\sqrt{\pi}},
$$

we can write the series in (34) as the following very-well-poised hypergeometric series:

$$
\begin{aligned}
& \widetilde{h}_{n}=\frac{\Gamma(3 n+3) \Gamma(n+1)^{3} \Gamma\left(n+\frac{1}{2}\right)^{2} \Gamma\left(n+\frac{3}{2}\right)^{2}}{\pi \Gamma(2 n+2)^{3} \Gamma\left(2 n+\frac{3}{2}\right)^{2}}
\end{aligned}
$$

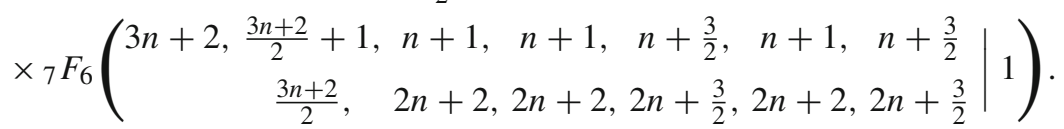


Applying now Bailey's transformation (see [17, Eq. (4.7.1.3)] or [22, Proposition 2]), after a little reduction of the gamma factors we get the Barnes-type integral

$$
\widetilde{h}_{n}=\frac{2 n+1}{2 \pi} \cdot \frac{1}{2 \pi i} \int_{-i \infty}^{i \infty} \frac{\Gamma(n+1+\xi)^{2} \Gamma\left(n+\frac{1}{2}+\xi\right) \Gamma\left(n+\frac{3}{2}+\xi\right) \Gamma(-\xi) \Gamma\left(-\frac{1}{2}-\xi\right)}{\Gamma(2 n+2+\xi) \Gamma\left(2 n+\frac{3}{2}+\xi\right)} \mathrm{d} \xi
$$

where the path separates the decreasing sequence of poles $\xi=-n-\frac{1}{2},-n-1,-n-$ $\frac{3}{2}, \ldots$ and the increasing sequence of poles $\xi=0, \frac{1}{2}, 1, \ldots$ of the integrand. The result can be expressed as a triple real integral thanks to a theorem of Nesterenko [22, Proposition 1]:

$$
\widetilde{h}_{n}=\frac{2 n+1}{2 \pi} \iiint_{[0,1]^{3}} \frac{x^{n}(1-x)^{n} y^{n-1 / 2}(1-y)^{n} z^{n+1 / 2}(1-z)^{n-1 / 2}}{(1-(1-x y) z)^{n+1}} \mathrm{~d} x \mathrm{~d} y \mathrm{~d} z
$$

On the other hand, using the duplication formula $\Gamma(z) \Gamma\left(z+\frac{1}{2}\right)=\sqrt{\pi} 2^{1-2 z} \Gamma(2 z)$ and the Barnes-type and Euler integrals for the Gauss hypergeometric function (see [17, Sections 1.6.1 and 4.1]) we can transform (35) further:

$$
\begin{aligned}
\widetilde{h}_{n} & =4(2 n+1) \cdot \frac{1}{2 \pi i} \int_{-i \infty}^{i \infty} \frac{\Gamma(2 n+1+2 \xi) \Gamma(2 n+2+2 \xi) \Gamma(-1-2 \xi)}{\Gamma(4 n+3+2 \xi)} \mathrm{d} \xi \\
& =2(2 n+1) \cdot \frac{1}{2 \pi i} \int_{-i \infty}^{i \infty} \frac{\Gamma(2 n+\xi) \Gamma(2 n+1+\xi) \Gamma(-\xi)}{\Gamma(4 n+2+\xi)} \mathrm{d} \xi \\
& =2(2 n+1) \frac{\Gamma(2 n) \Gamma(2 n+1)}{\Gamma(4 n+2)}{ }_{2} F_{1}\left(\begin{array}{c}
2 n, \\
2 n+1 \\
4 n+2
\end{array} \mid-1\right)=2 \int_{0}^{1} \frac{x^{2 n-1}(1-x)^{2 n+1}}{(1+x)^{2 n+1}} \mathrm{~d} x,
\end{aligned}
$$

and for the latter integral the decomposition

$$
\widetilde{h}_{n}=\widetilde{a}_{0, n}+\widetilde{a}_{1, n} \log 2 \quad \text { with } \quad d_{2 n} \widetilde{a}_{0, n} \in \mathbb{Z} \quad \text { and } \quad \widetilde{a}_{1, n} \in \mathbb{Z}
$$

is known (see, for example, [19]). The arithmetic inclusions in (37) are much better than the ones we have from Proposition 4; this suggests the existence of a 'power denominator conjecture' for the linear forms constructed in Sect. 3.3. In addition, a more general form of the triple integral in (36) could be of use in study of the quality of rational approximations to $\log 2$; it is due to a remarkable resemblance of such integrals with the ones used by Rhin and Viola [15] in proving the record irrationality measure for $\zeta$ (3). For a different construction of rational approximations to $\log 2$ using the Rhin-Viola method, we refer the reader to the paper [13], where Marcovecchio obtains a new irrationality measure for this constant. 
Open Access This article is distributed under the terms of the Creative Commons Attribution Noncommercial License which permits any noncommercial use, distribution, and reproduction in any medium, provided the original author(s) and source are credited.

\section{References}

1. Apéry, R.: Irrationalité de $\zeta(2)$ et $\zeta(3)$. Astérisque 61, 11-13 (1979)

2. Ball, K., Rivoal, T.: Irrationalité d'une infinité de valeurs de la fonction zêta aux entiers impairs. Invent. Math. 146(1), 193-207 (2001)

3. Bedulev, E.V.: On the linear independence of numbers over number fields. Mat. Zametki 64(4), 506517 (1998); English transl., Math. Notes 64(3-4), 440-449 (1998)

4. Colmez, P.: Arithmétique de la fonction zêta. La fonction zêta, pp. 37-164 (Journées X-UPS 2002)

5. Davenport, H., Schmidt, W.M.: Approximation to real numbers by quadratic irrationals. Acta Arith. 13, 169-176 (1967)

6. Davenport, H., Schmidt, W.M.: Approximation to real numbers by algebraic integers. Acta Arith. 15, 393-416 (1969)

7. Fischler, S.: Irrationalité de valeurs de zêta (d'après Apéry, Rivoal, ...). Séminaire Bourbaki 20022003, exp. no. 910. Astérisque 294, 27-62 (2004)

8. Fischler, S.: Restricted rational approximation and Apéry-type constructions. Indag. Math. (2010, in press)

9. Fischler, S., Rivoal, T.: Irrationality exponent and rational approximations with prescribed growth. Proc. Am. Math. Soc. (2010, in press)

10. Jouhet, F., Mosaki, E.: Irrationalité aux entiers impairs positifs d'un $q$-analogue de la fonction zêta de Riemann. Int. J. Number Theory (2007, in press). arXiv:0712.1762 [math.CO]

11. Krattenthaler, C., Rivoal, T.: Hypergéométrie et fonction zêta de Riemann. Mem. Am. Math. Soc. 186(875) (2007)

12. Krattenthaler, C., Rivoal, T., Zudilin, W.: Séries hypergéométriques basiques, $q$-analogues des valeurs de la fonction zêta et séries d'Eisenstein. J. Inst. Math. Jussieu 5(1), 53-79 (2006)

13. Marcovecchio, R.: The Rhin-Viola method for $\log 2$. Acta Arith. 139(2), 147-184 (2009)

14. Nesterenko, Yu.V.: On the linear independence of numbers. Vestnik Moskov. Univ. Ser. I Mat. Mekh. 1, 46-49 (1985); English transl., Moscow Univ. Math. Bull. 40(1), 69-74 (1985)

15. Rhin, G., Viola, C.: The group structure for $\zeta$ (3). Acta Arith. 97(3), 269-293 (2001)

16. Rivoal, T.: La fonction zêta de Riemann prend une infinité de valeurs irrationnelles aux entiers impairs. C. R. Acad. Sci. Paris Sér. I Math. 331(4), 267-270 (2000)

17. Slater, L.J.: Generalized Hypergeometric Functions. Cambridge University Press, Cambridge (1966)

18. Töpfer, T.: Über lineare Unabhängigkeit in algebraischen Zahlkörpern. Results Math. 25(1-2), 139152 (1994)

19. Viola, C.: Hypergeometric functions and irrationality measures. Analytic number theory (Kyoto, 1996). In: London Mathematical Society Lecture Note Series, vol. 247, pp. 353-360. Cambridge University Press, Cambridge (1997)

20. Zudilin, W.: On the irrationality of the values of the Riemann zeta function. Izv. Ross. Akad. Nauk Ser. Mat. 66(3), 49-102 (2002); English transl., Izv. Math. 66(3), 489-542 (2002)

21. Zudilin, W.: On the irrationality measure for a $q$-analogue of $\zeta(2)$. Mat. Sb. 193(8), 49-70 (2002); English transl., Sb. Math. 193(7-8), 1151-1172 (2002)

22. Zudilin, W.: Arithmetic of linear forms involving odd zeta values. J. Théor. Nombres Bordeaux 16(1), 251-291 (2004) 